\title{
Endoscopic Ultrasonography in the Diagnosis of Gastric Subepithelial Lesions
}

\author{
Eun Jeong Gong and Do Hoon Kim \\ Department of Gastroenterology, Asan Medical Center, University of Ulsan College of Medicine, Seoul, Korea
}

Subepithelial lesions occasionally found in the stomach of patients undergoing endoscopy may be either benign lesions or tumors with malignant potential. They may also appear due to extrinsic compression. Discrimination of gastric subepithelial lesions begins with meticulous endoscopic examination for size, shape, color, mobility, consistency, and appearance of the overlying mucosa. Accurate diagnosis can be achieved with endoscopic ultrasonography, which provides useful information on the exact size, layer-of-origin, and characteristic morphologic features to support a definitive diagnosis. Endoscopic ultrasonography also aids in the prediction of malignant potential, especially in gastrointestinal stromal tumors. Features of subepithelial lesions identified on endoscopic ultrasonography can be used to determine whether further diagnostic procedures such as endoscopic resection, fine needle aspiration, or core biopsy are required. Endoscopic ultrasonography is a valuable tool for diagnosis and clinical decision making during follow-up of gastric subepithelial lesions. Clin Endosc 2016;49:425-433

Key Words: Endosonography; Stomach neoplasms; Diagnosis

\section{INTRODUCTION}

A subepithelial lesion of the gastrointestinal tract is defined as an elevated lesion, mass, or bulge within the lumen that is usually covered by normal-appearing mucosa. Subepithelial lesions were previously described as submucosal tumors. However, subepithelial lesions may arise from any layer of the gastrointestinal wall and sometimes they are even an impression of surrounding structures outside the wall; therefore, the term "subepithelial lesion" is used more commonly. Subepithelial lesions are usually asymptomatic and incidentally observed during endoscopic examination; one early study reported the prevalence of endoscopically suspected gastric

Received: April 26, 2016 Revised: July 29, 2016

Accepted: July 29, 2016

Correspondence: Do Hoon Kim

Department of Gastroenterology, Asan Digestive Disease Research Institute, Asan Medical Center, University of Ulsan College of Medicine, 88 Olympic-ro 43-gil, Songpa-gu, Seoul 05505, Korea

Tel: +82-2-3010-3193, Fax: +82-2-476-0824, E-mail: dohoon.md@gmail.com

(cc) This is an Open Access article distributed under the terms of the Creative Commons Attribution Non-Commercial License (http://creativecommons.org/ licenses/by-nc/3.0) which permits unrestricted non-commercial use, distribution, and reproduction in any medium, provided the original work is properly cited. subepithelial lesions as $0.36 \% .{ }^{1}$ However, the diagnosis of subepithelial lesions is increasing owing to the widespread use of endoscopy for screening purposes, with an incidence of $0.76 \%$ reported in a recent study. ${ }^{2}$

Subepithelial lesions may be either benign lesions or tumors with malignant potential, such as gastrointestinal stromal tumors (GISTs) and carcinoid tumors. The differential diagnosis of subepithelial lesions is important, and it begins with meticulous endoscopic examination. The size, shape, color, mobility, consistency, pulsation, and presence of erosion or ulceration in the overlying mucosa should be evaluated. In general, subepithelial lesions have a normal-appearing mucosa; the presence of erythema or ulceration is associated with an increase in the size of the subepithelial lesion. ${ }^{3}$ A change in the shape or location of the lesion, mass, or bulge in response to changes in position or air volume may help in differentiating between extrinsic compression and intramural tumors. In addition, several signs that can be confirmed with biopsy forceps manipulation of the subepithelial lesions, including the rolling sign, pillow sign, naked fat sign, or tenting sign, can be useful in the differential diagnosis.

Improved evaluation of the gastric wall and surroundings 
Table 1. Characteristic Features of Gastric Subepithelial Lesions

\begin{tabular}{|c|c|c|}
\hline Type of lesion & EUS layer $^{\text {a) }}$ & EUS appearance \\
\hline Gastrointestinal stromal tumor & Fourth (rarely second) & $\begin{array}{l}\text { Hypoechoic (heterogeneous echogenicity, irregular margins, cystic spac- } \\
\text { es, and the presence of lymphadenopathy suggest malignancy) }\end{array}$ \\
\hline Leiomyoma & Second, fourth & Hypoechoic, well-circumscribed \\
\hline Lipoma & Third & Intensely hyperechoic, homogeneous \\
\hline Varices & Third & Anechoic, tubular, serpiginous \\
\hline Schwannoma & Fourth & Hypoechoic \\
\hline Inflammatory fibroid polyp & Second and/or third & Hypoechoic, homogeneous or mixed echogenicity, indistinct margins \\
\hline Heterotopic pancreas & Second, third, and/or fourth & Hypoechoic or mixed echogenic, anechoic ductal structure may be present \\
\hline Carcinoid & Second and/or third & Mildly hypoechoic or isoechoic, homogeneous \\
\hline Granular cell tumor & Second or third & Hypoechoic, homogeneous with smooth margins \\
\hline
\end{tabular}

EUS, endoscopic ultrasonography.

${ }^{a)}$ First layer (the interface of the luminal fluid and the mucosa), second layer (the deep mucosa), third layer (the submucosa), fourth layer (the muscularis propria), and fifth layer (the serosa or adventitia).
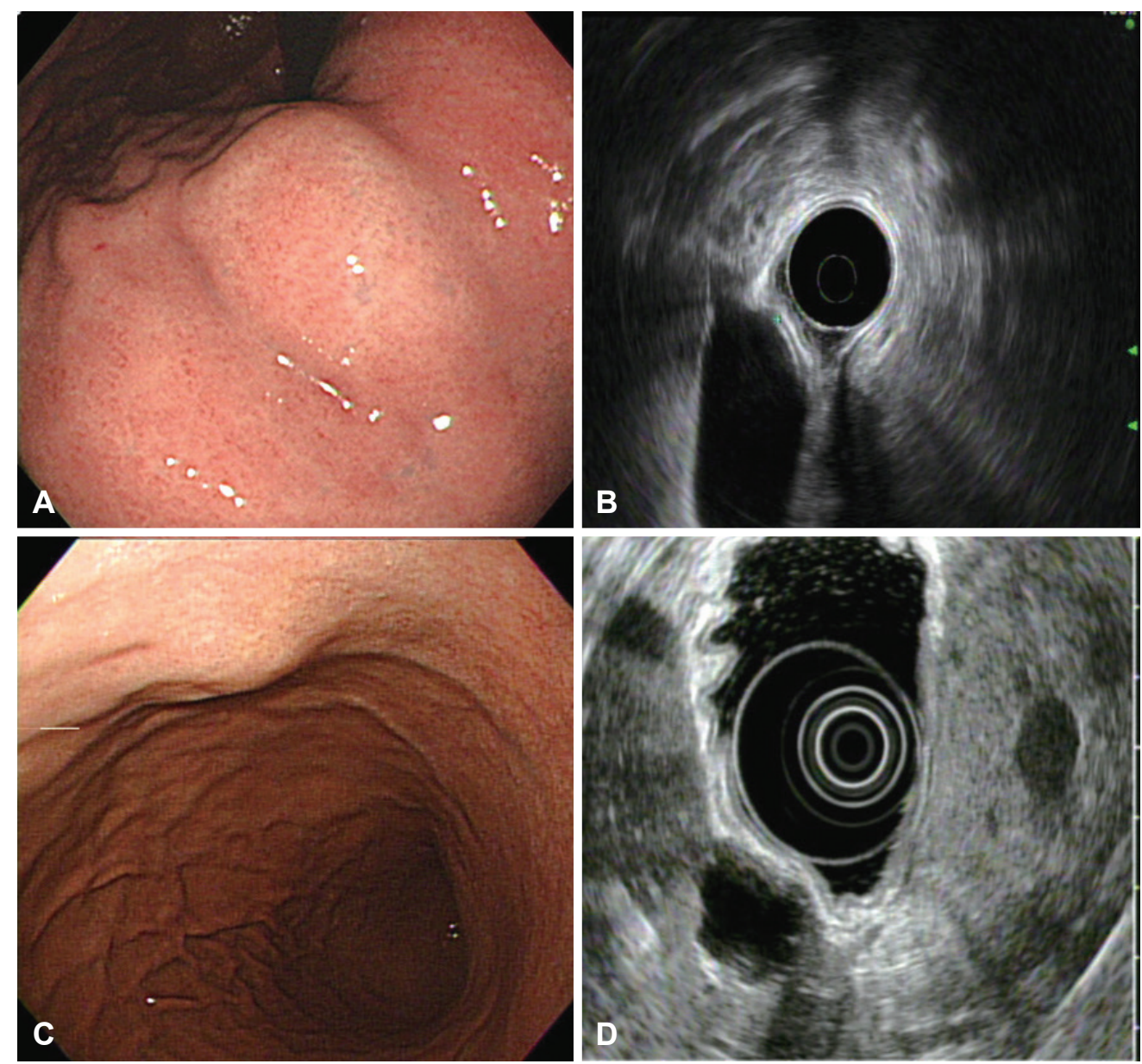

Fig. 1. Extramural lesion. (A) Bulge of the gastric body mimicking a subepithelial lesion. (B) Endoscopic ultrasonography showing intact gastric wall layers and compression by the gallbladder. (C) Subepithelial lesion in the gastric body. (D) Corresponding endoscopic ultrasonography image showing an anechoic cystic lesion of the liver.

has become possible with endoscopic ultrasonography (EUS). EUS can be used to approximate the size, layer-of-origin, margins, and echogenicity of the lesion, as well as to delineate its detailed morphology. EUS has particular value in differentiating intramural lesions from extrinsic compression, and it can help determine whether further diagnostic procedures are required. ${ }^{4,5}$ 

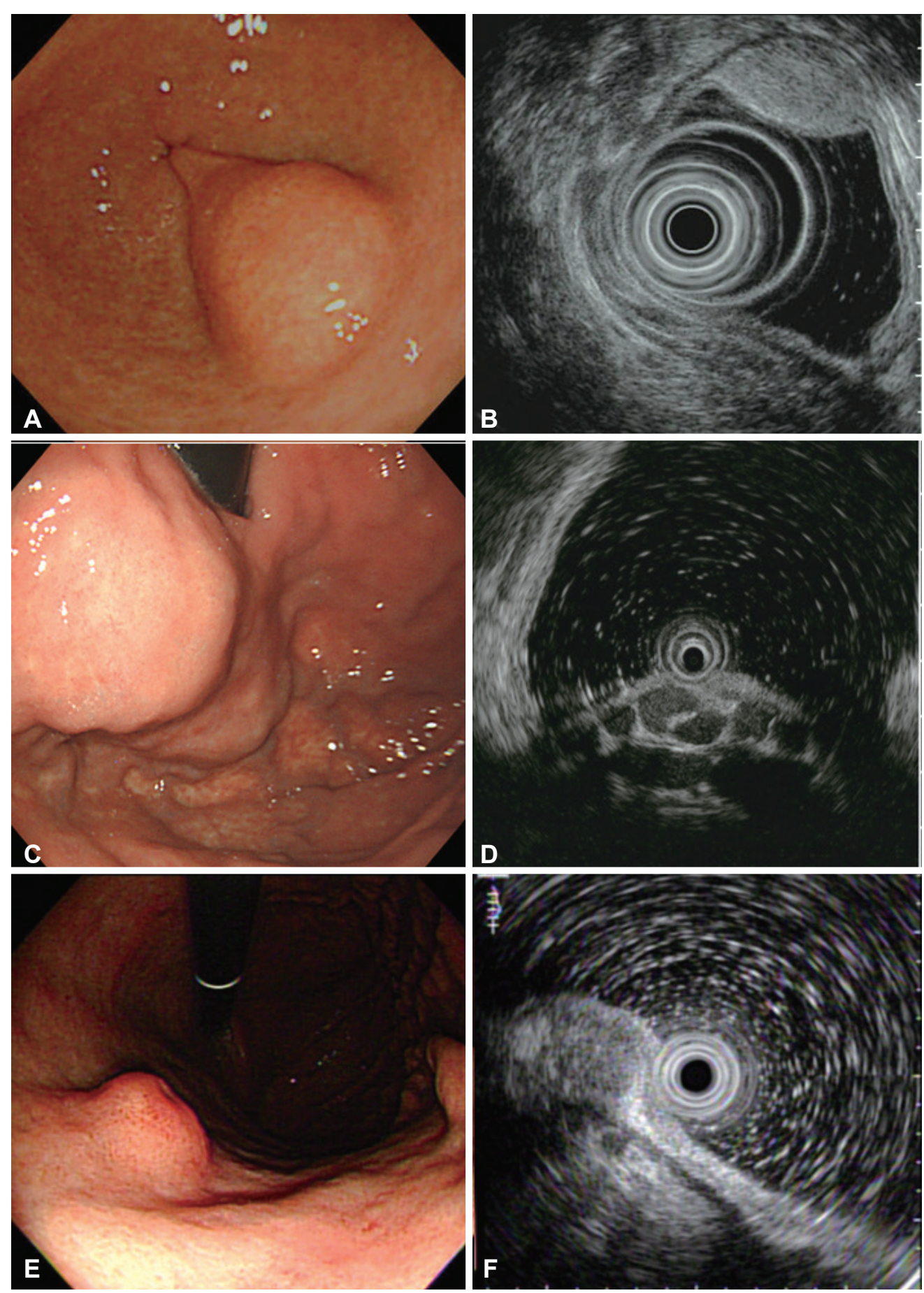

Fig. 2. Representative subepithelial lesions with typical features. (A, B) Gastric lipoma. (A) Endoscopic appearance of the lipoma located in the gastric antrum. (B) Endoscopic ultrasonography showing a hyperechoic, homogeneous mass arising from the third sonographic layer of the gastric wall. (C, D) Gastric varix. (C) Bulging, mass-like lesion in the gastric fundus and cardia. (D) Endoscopic ultrasonography showing anechoic, tubular, submucosal vascular structures. (E, F) Granular cell tumor. (E) Molar tooth-like, polypoid lesion in the gastric body. (F) Endosonographic image showing hypoechoic, homogeneous lesion with a smooth margin located in the second and third layers of the gastric wall.

\section{ENDOSONOGRAPHIC FEATURES}

The features observed during EUS are key to a correct diagnosis. EUS of the gastric wall reveals five distinct layers: the first layer is the interface echo between the superficial mucosa and the acoustic coupling medium; the second layer is the deep mucosa; the third layer is the submucosal layer; the fourth layer is the muscularis propria; and the fifth layer comprises 

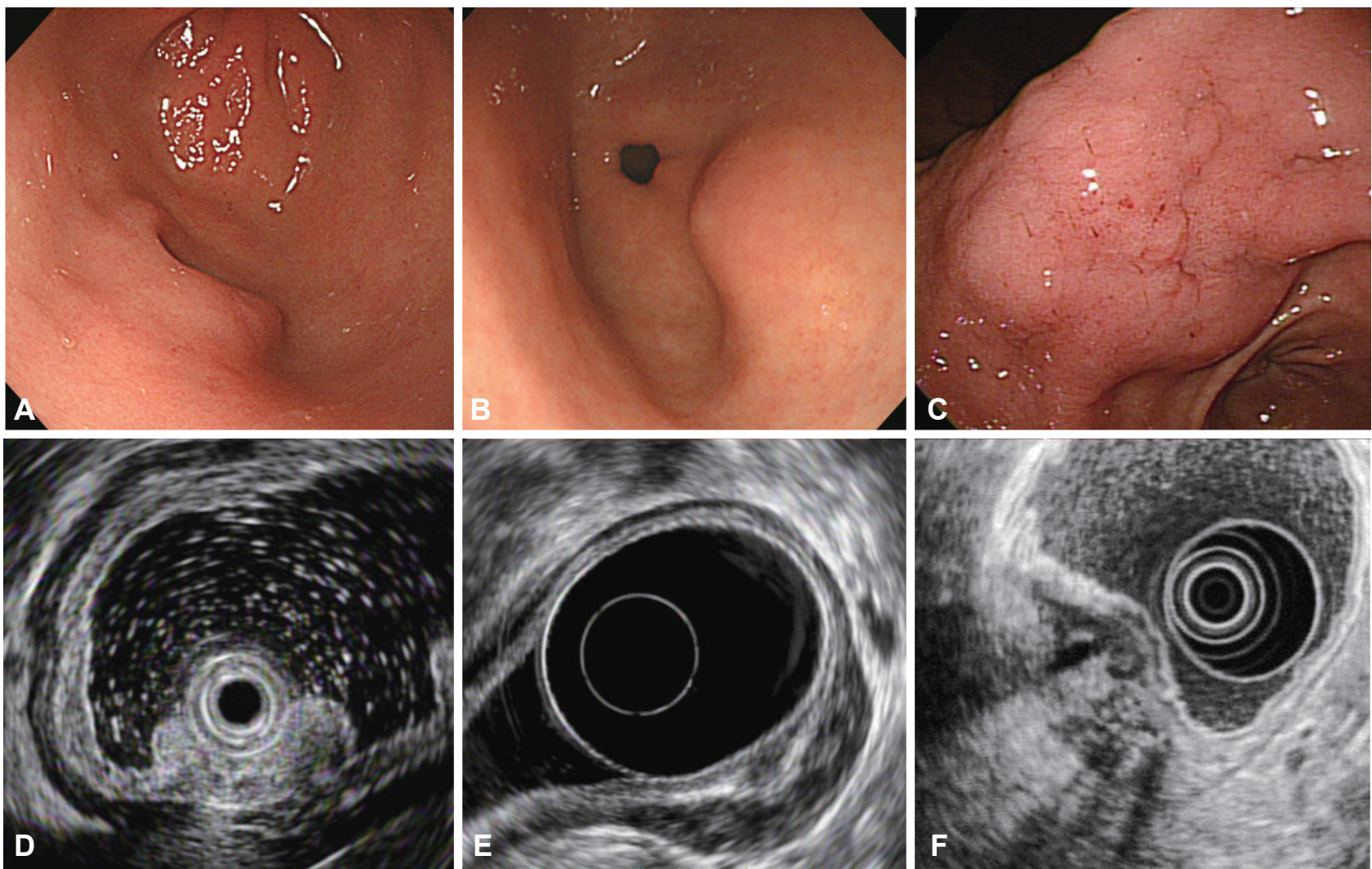

Fig. 3. Heterotopic pancreas. (A-C) Endoscopic findings of heterotopic pancreas located in the gastric antrum. (D-F) Corresponding endoscopic ultrasonography images showing hypoechoic lesions with internal anechoic ductal structures involving the third and fourth layers of the gastric wall.

the serosa and subserosal fat. During EUS examination, the layer-of-origin should be carefully defined. In addition, the size and morphologic pattern of the subepithelial lesion, including echogenicity, margins, and vascularity, should be evaluated. The characteristic details of subepithelial lesions are summarized in Table 1.

\section{Extramural lesions}

Intramural lesions may be difficult to differentiate from extrinsic compression using endoscopy alone; EUS may enable a more accurate discrimination. ${ }^{4-6}$ The most common sources of extrinsic gastric compression are the spleen and splenic vessels. ${ }^{4,5,7}$ Other sources include abdominal structures such as the liver, gallbladder, colon, and pancreas. In addition, pathologic conditions such as tumors, cysts, aneurysms, and enlarged lymph nodes may appear as gastric subepithelial lesions on endoscopy. ${ }^{6}$ During EUS, integrity of the normal gastric wall layer and the relationship of the lesion with adjacent organs should be observed (Fig. 1). The location of the lesion also informs the differential diagnosis: the spleen usually makes an impression in the gastric fundus and upper body, whereas the gallbladder compresses the gastric antrum.

\section{Intramural lesions}

\section{Lipomas}

Lipomas are benign, slow-growing tumors composed of mature lipocytes. On endoscopy, they usually present as soft, solitary lesions with a yellowish color and they often exhibit a pillow (or cushion) sign when pressed with biopsy forceps. ${ }^{4}$ Intensely hyperechoic, homogeneous, well-circumscribed lesions arising from the third layer of the gastric wall are unique EUS features of lipomas. If these features are observed, no further evaluation is needed (Fig. 2A, B). ${ }^{4}$

\section{Heterotopic pancreas}

Heterotopic pancreas is defined as ectopic pancreatic tissue with no anatomic or vascular connection to the pancreas. It is typically located in the gastric antrum and may have central umbilication. Heterotopic pancreas commonly arises from the submucosal layer of the gastric wall, but lesions may involve multiple layers (second, third, and/or fourth layers) with disruption of the layers. ${ }^{8}$ Hypoechoic or mixed echogenic lesions with internal structures suggesting ducts or anechoic microcysts are features indicative of heterotopic pancreas (Fig. 3). 

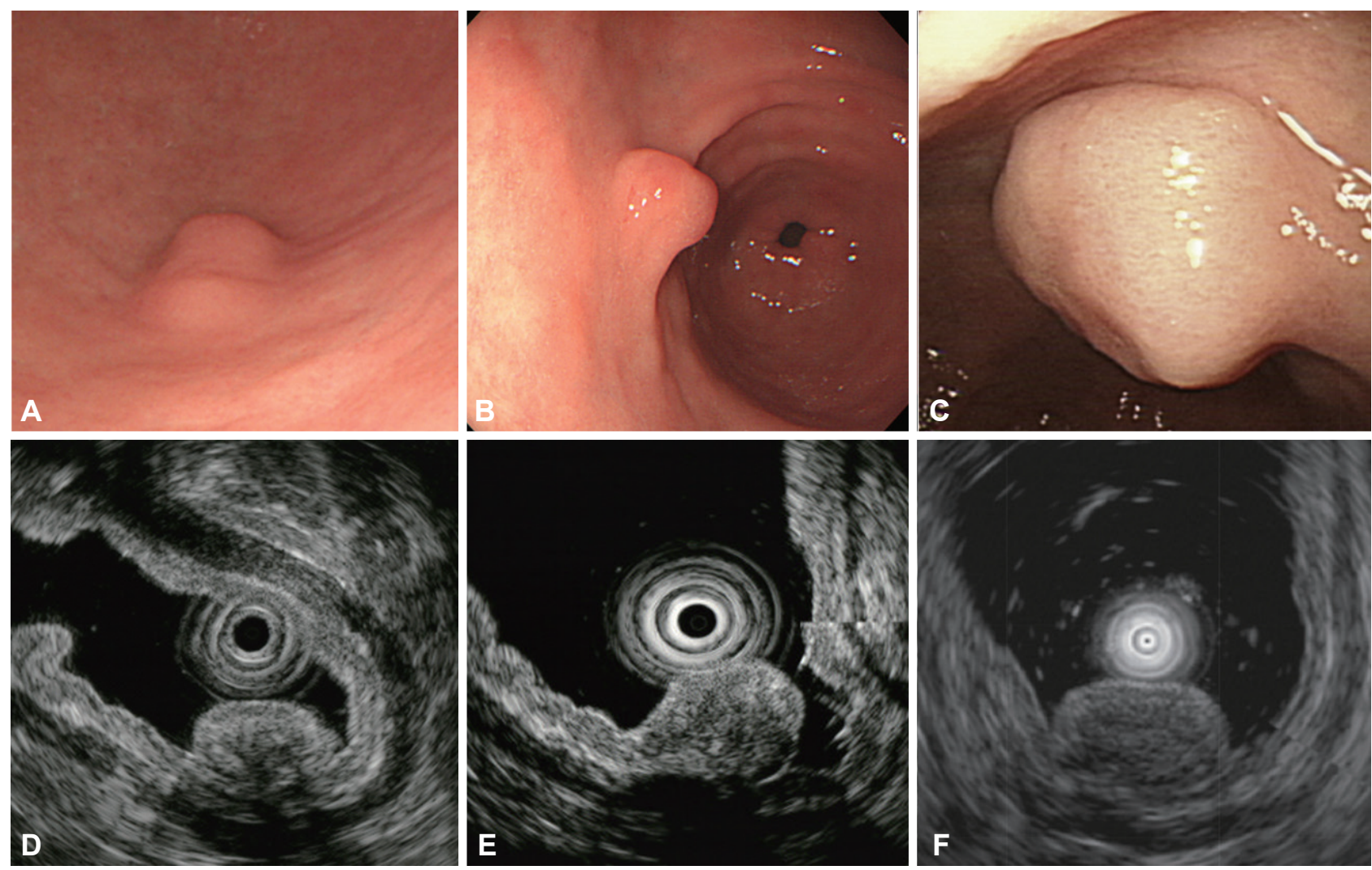

Fig. 4. Gastric inflammatory fibroid polyp. (A-C) Endoscopic images showing a small, polypoid lesion in the gastric antrum. (D-F) Endoscopic ultrasonography showing a hypoechoic lesion with indistinct margins located in the second layer of the gastric wall.

\section{Inflammatory fibroid polyps}

Gastric inflammatory fibroid polyps are rare, benign lesions presenting as semipedunculated protrusions with occasional erythematous depression, ulceration, or a white cap on their central surface. They are located in the antrum or prepyloric region. ${ }^{9}$ On EUS, inflammatory fibroid polyps are hypoechoic, homogeneous lesions with indistinct margins located in the second or third layer of the gastric wall, without involvement of the muscularis propria (Fig. 4). ${ }^{10}$

\section{Varices}

Gastric varices appear as hypoechoic or anechoic, tubular, or serpiginous structures with smooth margins located in the submucosal layer of the gastric wall, accompanied by perigastric collateral vessels (Fig. 2C, D). They can be differentiated from submucosal cysts according to the patient's medical history, the shape of the structure, and if the structure can be easily compressed. Doppler examination can demonstrate flow within the structure, which is a definitive diagnostic clue.

\section{Carcinoid tumors}

Gastric carcinoid tumors are neuroendocrine tumors that originate from enterochromaffin-like cells. These slow-grow- ing tumors with malignant potential arise from the mucosal layer of the gastric wall and can invade into deeper structures. ${ }^{11,12}$ On endoscopy, carcinoid tumors appear as small, round, sessile, or polypoid lesions with occasional central erythematous depression or ulceration. On EUS, carcinoids are mildly hypoechoic or isoechoic, homogeneous lesions with distinct smooth margins located in the second or third layer of the gastric wall (Fig. 5). Carcinoid tumors can be diagnosed by endoscopic biopsy even when the overlying mucosa appears normal, whereas the yield of mucosal biopsies from other subepithelial lesions is usually low.

\section{Leiomyomas}

Leiomyomas are benign tumors that arise from either the muscularis mucosa or the muscularis propria. The location of the lesion may be important in the clinical diagnosis as leiomyoma are frequently found in the esophagus, but rarely occur in the stomach. ${ }^{13}$ These tumors present as hypoechoic, homogeneous, well-circumscribed lesions in the second or fourth layer of the gastric wall on EUS examination (Fig. 6A, B).

\section{Gastrointestinal stromal tumors}

GISTs are the most commonly identified mesenchymal 

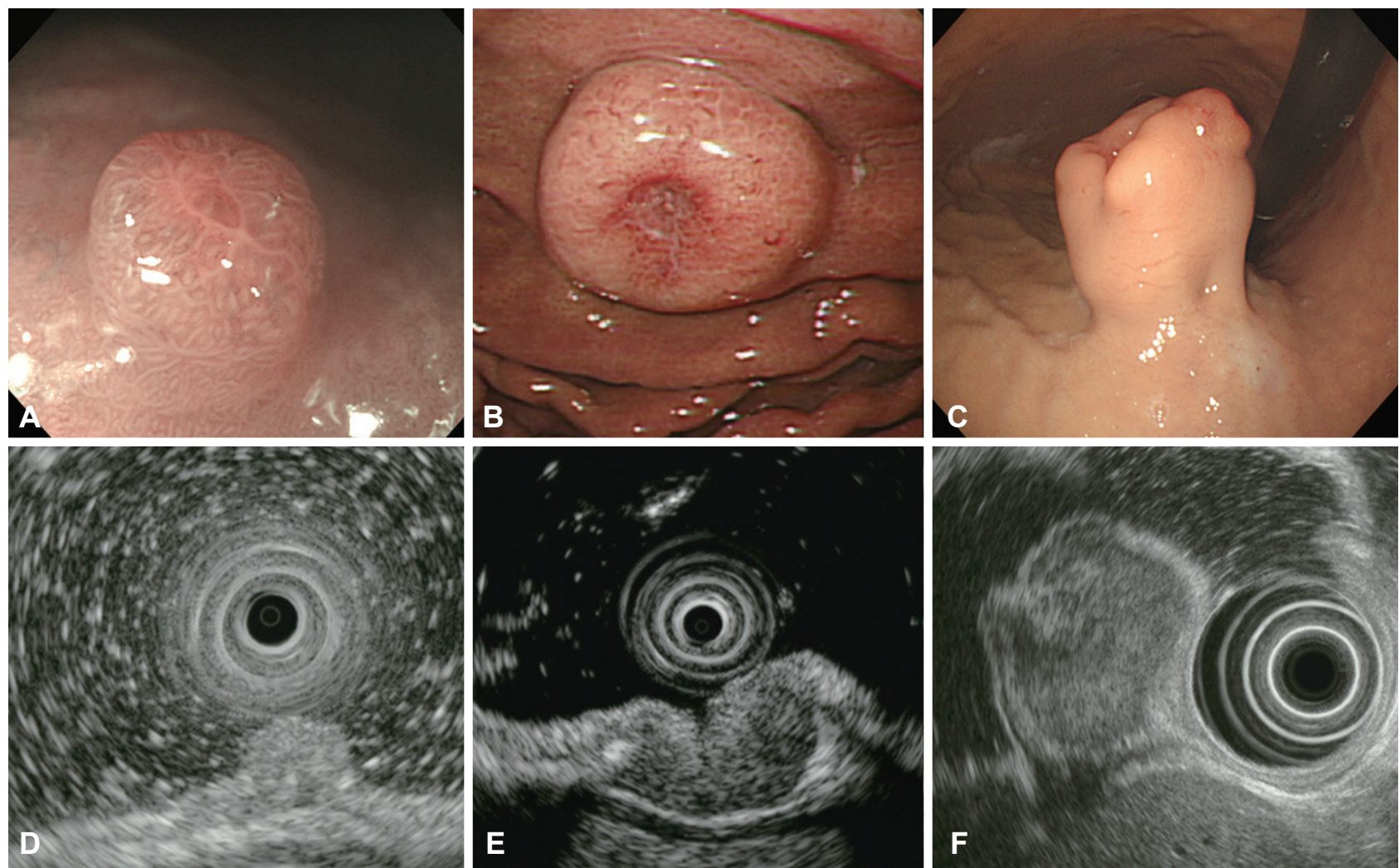

Fig. 5. Gastric carcinoid tumor. (A-C) Endoscopic images of a small, round, polypoid lesion with central umbilication in the gastric body. (D-F) Endoscopic ultrasonography showing a hypoechoic lesion with indistinct margins located in the second and third layers of the gastric wall.

tumors in the stomach and have malignant potential. ${ }^{14}$ GISTs arise from the interstitial cells of Cajal and can be identified using immunohistochemical staining for $\mathrm{CD} 117$, the protein product of the c-kit proto-oncogene. ${ }^{15}$ The risk of malignancy in GISTs varies widely and depends on tumor size and mitotic count. ${ }^{15}$ Endoscopically, GISTs appear as round or dumbbell-shaped lesions and occasionally present with central umbilication or ulceration. On EUS, they are generally hypoechoic, relatively homogeneous masses originating from either the second or fourth layer of the gastric wall (Fig. 6C, D). Many features observed on EUS, including size, irregular margins, intratumoral cystic spaces, presence of echogenic foci, and presence of peritumoral lymphadenopathy, are recognized as predictors of malignancy. ${ }^{16,17}$ Although the accuracy of EUS in the diagnosis of small subepithelial lesions is limited, this approach can be useful in differentiating GISTs from other benign tumors based on changes in echogenicity or an increase in size during follow-up. ${ }^{18,19}$ Nevertheless, none of these features are specific for the prediction of malignant potential, and histopathological diagnosis remains the gold standard.

\section{Schwannomas}

Schwannomas are benign S100 protein-positive spindle cell tumors usually located in the proximal part of the stomach. ${ }^{20}$
They appear as hypoechoic, heterogeneous tumors originating from the fourth layer of the gastric wall, and their endosonographic appearance is similar to that of leiomyomas or GISTs. Comparing the echogenicity of suspected lesions with that of the muscularis propria may be helpful for the differential diagnosis; the echogenicity of schwannomas is lower than that of the normal muscularis propria, whereas that of leiomyomas and GISTs is similar or higher (Fig. $6 \mathrm{E}, \mathrm{F}$ ). ${ }^{21-23}$

\section{Glomus tumor}

Glomus tumors are mesenchymal tumors originating from modified smooth muscle cells of the glomus body. These tumors are commonly observed in the dermis or subcutis but are rarely found in the gastrointestinal tract. Most gastric glomus tumors are benign. On EUS, they usually appear as well-circumscribed, hypoechoic masses with an internal heterogeneous echo pattern mixed with hyperechogenic spots located in the third and/or fourth layer of the gastric wall. ${ }^{24,25}$ Although EUS may help identify the layer-of-origin, there are no specific EUS findings to establish a diagnosis of glomus tumor. $^{26}$

\section{Granular cell tumor}

Granular cell tumors are rare lesions of neural origin that 

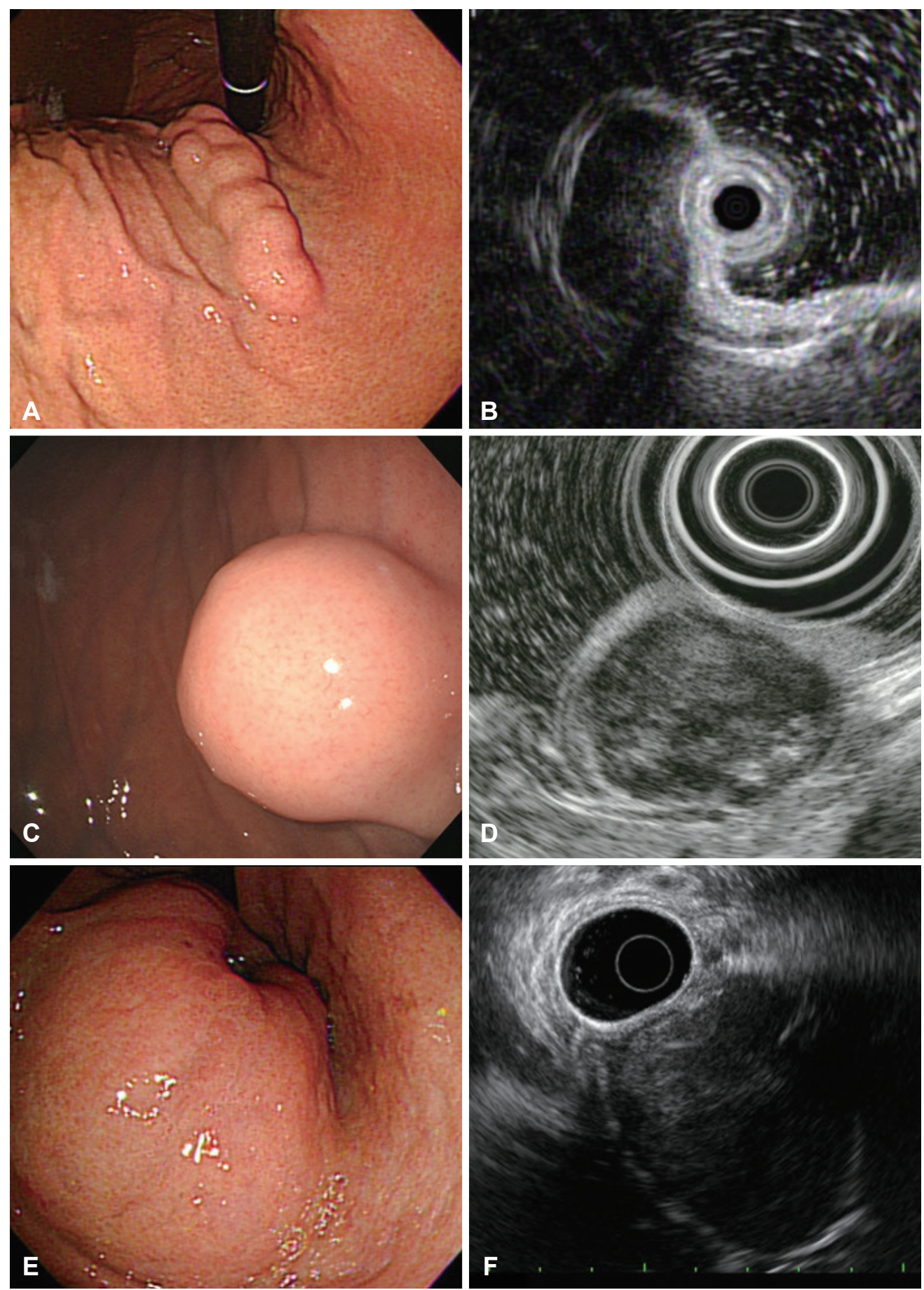

Fig. 6. Hypoechoic lesions arising from the muscularis propria. Endoscopic and corresponding endosonographic images of a (A, B) leiomyoma, $(C, D)$ gastrointestinal stromal tumor and $(E, F)$ schwannoma.

are most commonly found in the esophagus, but rarely in the stomach. ${ }^{27,28}$ Granular cell tumors arising in the stomach are generally considered benign, and only a small number of cases are malignant. ${ }^{29}$ Endoscopically, granular cell tumors are small (usually less than $20 \mathrm{~mm}$ in diameter), sessile nodules or polyps with a yellowish-white color resembling molar teeth and, when they occur in the stomach, they are most common- ly located in the proximal stomach. ${ }^{28,30}$ On EUS, they appear as hypoechoic, homogeneous lesions with smooth margins arising from the second or third layer of the gastric wall (Fig. $2 \mathrm{E}, \mathrm{F})$.

\section{Malignancy mimicking a subepithelial lesion}

Malignant tumors such as adenocarcinomas, lymphomas, 
and metastatic carcinomas can present as subepithelial lesions. Endoscopic and EUS features of these lesions are not diagnostic, even though they have been previously reported as such, and thus histopathologic diagnosis is necessary. ${ }^{31-33}$ Gastric carcinomas mimicking subepithelial lesions are rare and tend to have irregular circumferential margins where the tumor is elevated. On EUS, they are usually well-demarcated masses originating from the third layer of the gastric wall. ${ }^{31,32}$ Gastric lymphomas may appear as subepithelial lesions with various features such as erosion and ulceration. On EUS, lymphomas appear as hypoechoic, homogeneous lesions located between the second and fourth layers with layer disruption, and the presence of adjacent lymphadenopathy can be helpful in their diagnosis. The most frequent primary tumors with gastrointestinal metastasis are breast carcinoma, melanoma, and lung cancer. ${ }^{33}$ On EUS, metastatic carcinomas appear as hypoechoic, heterogeneous lesions in any or all of the EUS layers of the gastric wall. ${ }^{33}$

\section{CONCLUSIONS}

Gastric subepithelial lesions are relatively common features encountered during endoscopic examinations. EUS can be used to determine the size, layer-of-origin, margins, echogenicity, and the detailed morphology of these lesions. In addition, the features identified on EUS can be used to determine the need for further diagnostic procedures such as endoscopic resection, fine needle aspiration, or core biopsy. Extramural lesions or benign subepithelial lesions, such as lipomas, do not require further management or follow-up. Endoscopic resection can be considered a diagnostic or therapeutic approach for small lesions originating from the muscularis mucosa or the submucosal layer. EUS-guided fine needle aspiration or core biopsy can be useful in the diagnosis of subepithelial lesions larger than $2 \mathrm{~cm}$ in size or those with clinically malignant features. ${ }^{34}$ Although histopathological diagnosis remains the gold standard, EUS is useful in the differential diagnosis of gastric subepithelial lesions and can guide further management and follow-up.

\section{Conflicts of Interest}

The authors have no financial conflicts of interest.

\section{REFERENCES}

1. Hedenbro JL, Ekelund M, Wetterberg P. Endoscopic diagnosis of submucosal gastric lesions. The results after routine endoscopy. Surg Endosc 1991;5:20-23.

2. Lim YJ, Son HJ, Lee JS, et al. Clinical course of subepithelial lesions detected on upper gastrointestinal endoscopy. World J Gastroenterol
2010;16:439-444.

3. Song JH, Kim SG, Chung SJ, Kang HY, Yang SY, Kim YS. Risk of progression for incidental small subepithelial tumors in the upper gastrointestinal tract. Endoscopy 2015;47:675-679.

4. Hwang JH, Saunders MD, Rulyak SJ, Shaw S, Nietsch H, Kimmey MB. A prospective study comparing endoscopy and EUS in the evaluation of GI subepithelial masses. Gastrointest Endosc 2005;62:202-208.

5. Rosch T, Kapfer B, Will U, et al. Accuracy of endoscopic ultrasonography in upper gastrointestinal submucosal lesions: a prospective multicenter study. Scand J Gastroenterol 2002;37:856-862.

6. Oztas E, Oguz D, Kurt M, et al. Endosonographic evaluation of patients with suspected extraluminal compression or subepithelial lesions during upper gastrointestinal endoscopy. Eur J Gastroenterol Hepatol 2011;23:586-592.

7. Motoo Y, Okai T, Ohta $\mathrm{H}$, et al. Endoscopic ultrasonography in the diagnosis of extraluminal compressions mimicking gastric submucosal tumors. Endoscopy 1994;26:239-242.

8. Matsushita M, Hajiro K, Okazaki K, Takakuwa H. Gastric aberrant pancreas: EUS analysis in comparison with the histology. Gastrointest Endosc 1999;49(4 Pt 1):493-497.

9. Matsushita M, Okazaki K. Characteristic endoscopic features of gastric inflammatory fibroid polyps. J Gastroenterol Hepatol 2005;20:1310.

10. Matsushita M, Hajiro K, Okazaki K, Takakuwa H. Gastric inflammatory fibroid polyps: endoscopic ultrasonographic analysis in comparison with the histology. Gastrointest Endosc 1997;46:53-57.

11. Soga J. Early-stage carcinoids of the gastrointestinal tract: an analysis of 1914 reported cases. Cancer 2005;103:1587-1595.

12. Nakamura S, Iida M, Yao T, Fujishima M. Endoscopic features of gastric carcinoids. Gastrointest Endosc 1991;37:535-538.

13. Miettinen M, Lasota J. Gastrointestinal stromal tumors: definition, clinical, histological, immunohistochemical, and molecular genetic features and differential diagnosis. Virchows Arch 2001;438:1-12.

14. Miettinen M, Sarlomo-Rikala M, Lasota J. Gastrointestinal stromal tumors: recent advances in understanding of their biology. Hum Pathol 1999;30:1213-1220.

15. Fletcher CD, Berman JJ, Corless C, et al. Diagnosis of gastrointestinal stromal tumors: a consensus approach. Hum Pathol 2002;33:459-465.

16. Palazzo L, Landi B, Cellier C, Cuillerier E, Roseau G, Barbier JP. Endosonographic features predictive of benign and malignant gastrointestinal stromal cell tumours. Gut 2000;46:88-92.

17. Shah P, Gao F, Edmundowicz SA, Azar RR, Early DS. Predicting malignant potential of gastrointestinal stromal tumors using endoscopic ultrasound. Dig Dis Sci 2009;54:1265-1269.

18. Reddymasu SC, Oropeza-Vail M, Pakseresht K, et al. Are endoscopic ultrasonography imaging characteristics reliable for the diagnosis of small upper gastrointestinal subepithelial lesions? J Clin Gastroenterol 2012;46:42-45

19. Gill KR, Camellini L, Conigliaro R, et al. The natural history of upper gastrointestinal subepithelial tumors: a multicenter endoscopic ultrasound survey. J Clin Gastroenterol 2009;43:723-726.

20. Voltaggio L, Murray R, Lasota J, Miettinen M. Gastric schwannoma: a clinicopathologic study of 51 cases and critical review of the literature. Hum Pathol 2012;43:650-659.

21. Okai T, Minamoto T, Ohtsubo K, et al. Endosonographic evaluation of c-kit-positive gastrointestinal stromal tumor. Abdom Imaging 2003; 28:301-307.

22. Jung MK, Jeon SW, Cho CM, et al. Gastric schwannomas: endosonographic characteristics. Abdom Imaging 2008;33:388-390.

23. Zhong DD, Wang $\mathrm{CH}, \mathrm{Xu} J \mathrm{H}$, Chen MY, Cai JT. Endoscopic ultrasound features of gastric schwannomas with radiological correlation: a case series report. World J Gastroenterol 2012;18:7397-7401.

24. Imamura A, Tochihara M, Natsui K, et al. Glomus tumor of the stomach: endoscopic ultrasonographic findings. Am J Gastroenterol 1994;89:271272.

25. Baek YH, Choi SR, Lee BE, Kim GH. Gastric glomus tumor: analysis of 
endosonographic characteristics and computed tomographic findings. Dig Endosc 2013;25:80-83.

26. Debol SM, Stanley MW, Mallery S, Sawinski E, Bardales RH. Glomus tumor of the stomach: cytologic diagnosis by endoscopic ultrasound-guided fine-needle aspiration. Diagn Cytopathol 2003;28:316321.

27. Morrison JG, Gray GF Jr, Dao AH, Adkins RB Jr. Granular cell tumors. Am Surg 1987;53:156-160.

28. Lack EE, Worsham GF, Callihan MD, et al. Granular cell tumor: a clinicopathologic study of 110 patients. J Surg Oncol 1980;13:301-316.

29. Matsumoto H, Kojima $\mathrm{Y}$, Inoue $\mathrm{T}$, et al. A malignant granular cell tumor of the stomach: report of a case. Surg Today 1996;26:119-122.

30. Ensari A. Granular cell tumor: what's new in diagnosis and treatment?
Turk J Gastroenterol 2007;18:135-138.

31. Kume K, Yoshikawa I, Yamazaki M, Abe S, Murata I, Otsuki M. A case of gastric cancer with features of submucosal tumor. Gastrointest Endosc 2001;53:247-249.

32. Hosoda Y, Hanai H, Arai H, et al. Early gastric cancer exhibiting the features of submucosal tumor: report of a case. Endoscopy 1999;31:S11.

33. Wiech T, Walch A, Werner M. Histopathological classification of nonneoplastic and neoplastic gastrointestinal submucosal lesions. Endoscopy 2005;37:630-634.

34. Cho JW; Korean ESD Study Group. Current guidelines in the management of upper gastrointestinal subepithelial tumors. Clin Endosc 2016;49:235-240. 